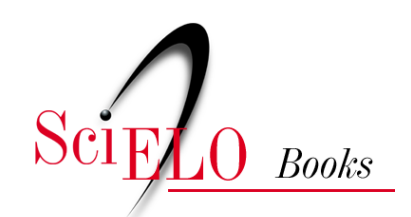

\title{
Capítulo VII - Clasificación automática de nódulos mamográficos basada en fusión de información visual multi-vista
}

\author{
Fabián Narváez
}

\section{SciELO Books / SciELO Livros / SciELO Libros}

NARVÁEZ, F. Clasificación automática de nódulos mamográficos basada en fusión de información visual multi-vista. In: INGA ORTEGA, E., ed. Aplicaciones e innovación de la ingeniería en ciencia y tecnología [online]. Quito: Editorial Abya-Yala, 2019, pp. 233-264. ISBN: 978-9978-10-491-0. https://doi.org/10.7476/9789978104910.0009.

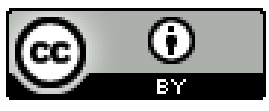

All the contents of this work, except where otherwise noted, is licensed under a Creative Commons Attribution 4.0 International license.

Todo o conteúdo deste trabalho, exceto quando houver ressalva, é publicado sob a licença Creative Commons Atribição 4.0.

Todo el contenido de esta obra, excepto donde se indique lo contrario, está bajo licencia de la licencia $\underline{\text { Creative }}$ Commons Reconocimento 4.0 . 


\title{
CAPÍTULO VII
}

\section{Clasificación automática de nódulos mamográficos basada en fusión de información visual multi-vista}

\section{Automatic classification of mammographic nodules based on multi-view information fusion}

\author{
Fabián Narváez* \\ Universidad Politécnica Salesiana Ecuador \\ *email: fnarvaeze@ups.edu.ec
}

\section{Resumen}

Una correcta evaluación e interpretación mamográfica demanda un alto nivel de experticia del radiólogo observador, depende directamente de un adecuado análisis visual de los hallazgos y la correlación de las características radiológicas extraídas de diferentes proyecciones mamográficas. En este artículo se presenta un esquema de clasificación automática de nódulos contenidos en Regiones de Interés (RoIs), extraídos de dos diferentes proyecciones mamográficas (Medio Lateral Oblicua y CreaneoCaudal) obtenidas de la misma glándula mamaria, mediante un estrategia de fusión de información ipsilateral. Una vez que, el radiólogo especialista selecciona una Región de Interés en las dos proyecciones mamográficas, estas son caracterizadas mediante descomposiciones multi-resolución y multi-escala, para lo cual, cada RoI es proyectada sobre dos espacios diferentes definidos por los momentos de Zernike y la transformada Curvelet, respectivamente. Así esta información heterogénea es optimamente fusionada mediante una estrategia de aprendizaje de múltiples kernels (Multiple Kernel Learning) construida por un entrenamiento con máquinas de soporte vectorial (SVM). El desempeño de la estrategia propuesta para clasificar nódulos malignos y benignos, fue evaluado respecto a un esquema de clasificación basado en el análisis de la RoI correspondiente a una única proyección, para lo cual se utilizaron un conjunto de 980 RoI extraídos de 490 casos de estudio de la base de datos de mamografía (DDSM) y 216 RoI extraídos de 108 casos de estudio de la base de datos INBreast. Los resulta- 
dos obtenidos reportan una sensibilidad del $98.3 \%$ y especificidad del $94.5 \%$ frente a una sensibilidad del $96.2 \%$ y especificidad del $93.1 \%$ obtenidos cuando se utilizaron características multi-resolución en una única proyección. Estos resultados sugieren que la estrategia propuesta puede ser útil en escenarios clínicos y puede contribuir a la capacitación de nuevos radiólogos como segundo lector.

\title{
Palabras clave
}

Diagnóstico asistido por computador, multiple kernel learning, transformada curvelet, momentos de zernike.

\begin{abstract}
Correct mammography assessment and interpretation demands great expertise of radiologist observer and depends directly on a suitable visual analysis of mammographic findings and their correlation with radiographic features extracted from different mammographic views. In this paper, an automatic classification scheme for mammographic nodules contained on Regions of Interest (ROIs) is presented, which is based on an information fusion approach by using RoIs extracted from two different mammographic views of the same breast, a Mediolateral Oblique (MLO) view and a craniocaudal (CC) view, respectively. Once the expert radiologist selects a RoI from the two mammographic projections, those are characterized by using a multiresolution and multiscale decomposition approaches. For doing so, each RoI is projected into two different spaces defined by Zernike moments and Curvelet transform, respectively. Finally, this extracted heterogeneous information is optimally fused by using a Multiple Kernel Learning strategy based on Support vector machine scheme. The performance of the herein proposed strategy, for classifying benign and malignant nodules, was evaluated respect to the classical mammographic analysis based on only mammographic view, for which a set of 980 ROIs extracted from 490 cases and other set of 216 RoI extracted from 108 cases, which were extracted from DDSM and INBreast databases, respectively. The obtained results reported a sensitivity of $98.3 \%$ and a specificity of $94.5 \%$ respect to $96.2 \%$ and $93.1 \%$ of sensibility and specificity, respectively, and obtained by the analysis based on an only mammographic view. These results suggest that the herein proposed strategy could be useful in real clinic scenarios and could be contributing to the training of new radiologists.
\end{abstract}

\section{Keywords}

Mammography, computer aided diagnosis, multiple kernel learning, curvelet transform, zernike moments.

\section{Introducción}

La mamografía es aún considerada la mejor técnica costo-efectiva para detectar anormalidades relacionadas a la presencia de cán- 
cer de mama en etapas tempranas de desarrollo $[1,2]$. El tamizaje a través de mamografías ha demostrado ser efectivo reduciendo la mortalidad por el cáncer de mama con porcentajes que varían desde el 30 al 70\% [3]. Sin embargo, la sensibilidad diagnóstica durante el tamizaje mamográfico es ampliamente afectada por: la calidad de las imágenes mamográficas, la similitud de los tejidos normales frente a los anormales visualizados en la mamografía y la experiencia del radiólogo observador, lo que produce una alta variabilidad inter e intra-observador durante la interpretación de los hallazgos mamográficos. Estudios previos han reportado que la sensibilidad del tamizaje mamográfico varía entre el $85 \%$ y 90\%, mientras que otros estudios han encontrado un importante porcentaje de presencia de falsos positivos durante los procesos diagnósticos, entre el $10 \%$ y $30 \%[4,5,6]$.

Durante las últimas décadas han sido propuestas diferentes soluciones con el propósito de incrementar la exactitud durante la interpretación mamográfica, su sensibilidad y especificidad, así como, reducir las tasas de biopsias innecesarias causadas por ciertas imprecisiones diagnósticas, entre estas soluciones importantes, el Colegio Americano de Radiología (American College of Radiology, ACR) desarrolló un sistema para el reporte diagnóstico mediante una terminología estandarizada para los informes de hallazgos anormales en imágenes mamarias (BI-RADS) mediante una semiología radiológica, para de esta manera asignar una categoría entre 0 y 6, de acuerdo a sus características radiológicas específicas. Estas categorías apoyan a los médicos y determinan la probabilidad de malignidad para considerar ciertas recomendaciones finales para el manejo y control de los hallazgos [7].

En el caso particular de las masas mamarias, de acuerdo al BI-RADS se describen por su forma, margen y densidad. En la quinta edición de BI-RADS, la forma se describe como ovalada, redonda e irregular y el margen como circunscrito, oscurecido, microlobulado, espiculado o indefinido. Finalmente, la densidad, a la cual se le considera como la atenuación de la masa con respecto al tejido glandular, se puede describir como Alta densidad, Igualdad de densidad, Baja densidad o Grasa. La figura 1 ilustra el espectro de características visuales que determinan el nivel de probabilidad de 
malignidad de acuerdo a la formas de masa y márgenes descritos en el sistema BI-RADS.

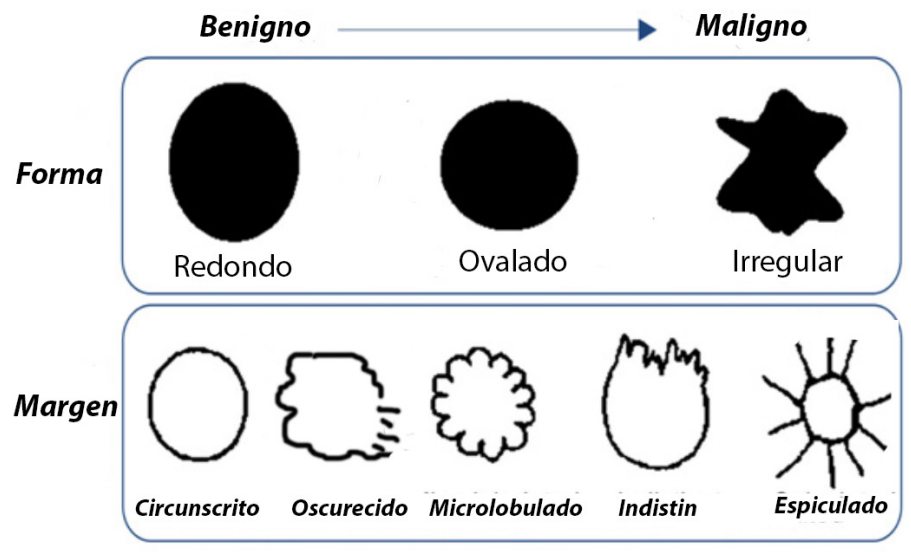

Figura 1. Diferentes tipo de formas y márgenes de masa definidos en el BIRADS. El grado de malignidad depende de estas características morfológicas

Como ilustración, una masa con forma redonda u ovalada, margen circunscrito y baja densidad tiene una alta probabilidad de ser benigna, mientras que una masa con forma irregular, margen espiculado y alta densidad es sospechosa de cáncer [8]. Este valor también define el manejo particular del paciente, es decir, los valores bajos implican que el paciente es mantenido con un adecuado control periódico mientras que los valores altos generalmente conducen a la posibilidad de biopsia y estudio patológico adicional. Sin embargo, la interpretación mamográfica sigue siendo una tarea subjetiva y difícil para el radiólogo.

Por otro lado, se han desarrollado tecnologías de Diagnóstico Asistido por Computador (CAD) como herramientas computacionales para asistir al radiólogo durante la toma de decisiones y son usadas como segundos lectores. En la actualidad, estos sistemas CAD son considerados como un potencial soporte al diagnóstico y tamizaje mamográfico, los mismos que han demostrado su utilidad durante la identificación de agrupamientos de micro-calcificaciones $[9,10]$, reportando una sensibilidad que varía entre el 75 al 90\%, 
pero su desempeño para soportar el diagnóstico de masas (nódulos) han sido reportados muy bajos, con una sensibilidad que varía entre el 54 al 72\% [11, 12]. La baja sensibilidad de los CAD comerciales y sus altas tasas de presencia de falsos positivos han reducido la confianza del radiólogo en estos sistemas [12]. La mayoría de los CAD están diseñados para producir una opinión sobre la presencia o no de cáncer, pero su análisis se basa únicamente mediante el procesamiento de la información de una sola proyección mamográfica, pudiendo ser ésta, la proyección Medio Lateral Oblicua (MLO) o la proyección Cráneo-Cudal (CC) de la misma mama, mientras que el protocolo clínico en los escenarios reales, el flujo de trabajo del radiólogo durante la interpretación de las lesiones mamográficas se basa en el análisis del hallazgo obtenido en las dos proyecciones, MLO y CC, para así determinar las características relevantes patológicas de la lesión y emitir un adecuado diagnóstico. Esto debido a la alta variabilidad de las características radiológicas que pueden presentar los hallazgos anormales vistos en diferentes proyecciones. Debido a que los sistemas CAD solo analizan una proyección, el uso clínico de este tipo de herramientas sigue siendo muy limitado, ya que la evidencia no es concluyente y, a veces, incluso contradictoria. Varios investigadores han descubierto que los sistemas CAD pueden aumentar la detección de las etapas iniciales de la enfermedad [13], mientras que otros informaron que, en un entorno de detección bien establecido, el uso de dichos sistemas no ayudó significativamente a mejorar la detección del cáncer [12].

Por lo tanto, varios estudios han intentado mejorar el rendimiento de los sistemas CAD, especialmente en el caso de análisis de masas [9, 14]. En la literatura, se han reportado varios algoritmos automáticos para segmentación y caracterización de masas dirigidos a tareas de clasificación como benignas o malignas [15, 16, 14, 17], reportando resultados con valores de AUC, área bajo la curva ROC, de aproximadamente 0.8 al combinar diferentes tipos de características de bajo nivel [17]. Típicamente, los descriptores de bajo nivel aplicados en mamografías son construidos mediante la extracción de las características morfológicas y de textura de las lesiones mamarias, previamente seleccionadas por el radiólogo. Entre la gran cantidad de enfoques reportados en la literatura se encuentran algunos descripto- 
res basados en los momentos de Zernike, los cuales se han utilizado para caracterizar formas y márgenes de masa previamente segmentadas y contenidas en regiones de interés [18, 19]. Además, los momentos de Zernike se han usado ampliamente en diferentes tipos de aplicaciones como un descriptor general de forma [20, 21, 22, 23] en masas bien definidas [18]. Del mismo modo, se han utilizado una gran variedad de técnicas de procesamiento de imágenes basadas en información estadística, estructurales y espectrales para analizar mamogramas [24], incluido el análisis por matrices de coocurrencia [25], dimensiones fractales [26, 27], wavelets [28, 29], curvelets [30] y contourlets $[31,32]$, mostrando un rendimiento muy variable.

Todos estos enfoques abordan el problema de clasificar una masa como maligna o benigna. Sin embargo, en la mayoría de los sistemas $\mathrm{CAD}$ se ignoran las diferencias visuales que pueden presentar la misma masa vista en varias proyecciones por lo que han limitado su análisis a las características visuales únicas en una única proyección, las cuales son evaluadas de forma independiente. Por lo tanto, la información entre las múltiples proyecciones o vistas complementarias de la misma mama, desafortunadamente no es considerada. La figura 2 ilustra la diferencia entre las características visuales de una misma masa observada en diferentes proyecciones.

Este hecho presenta como resultado inexactitudes y una alta incidencia de tasas de falsos positivos (PF). Recientemente, se ha propuesto el uso de información de las dos proyecciones mamográficas para mejorar el rendimiento del CAD respecto a una sola proyección. Hasta ahora, los sistemas CAD tradicionales se utilizan para identificar regiones mamográficas sospechosas en cada proyección por separado, de las que se extraen las características morfológicas y de textura. Estas características se utilizan luego para determinar la correspondencia entre el par de regiones extraídas de las dos proyecciones de la mama, ya sea utilizando diferentes clasificadores discriminativos, como el análisis discriminante lineal (LDA) y el k-vecino más cercano (K-NN). Estos enfoques demostraron una capacidad para discriminar entre correspondencias verdaderas y falsas, reduciendo los falsos positivos reportados por el uso de un sistema CAD de una única proyección. Aunque el uso de información de múltiples proyecciones ha demostrado ser útil para mejorar la detec- 
ción de masas mamográficas, sin embargo, estos enfoques dependen en gran medida de una segmentación precisa de la lesión, un desafío difícil durante el análisis de masas con límites no definidos.

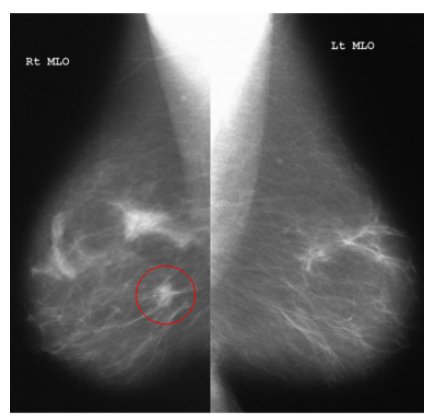

(a)

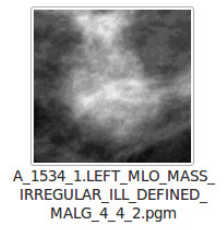

Lt ce

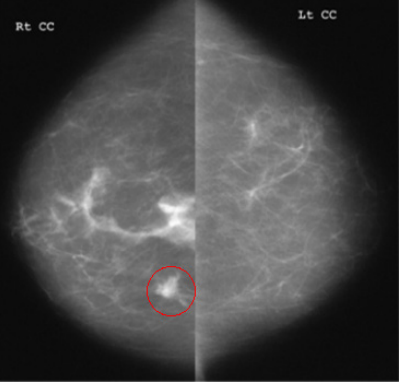

(b)

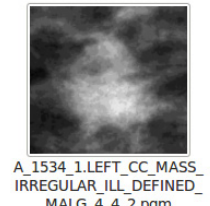

MALG 4 - 4 2.pgm

Figura 2. Caso de estudio mamográfico: a) Ilustra una RoI con una masa extraída de la proyección MLO de la mama izquierda b) Ilustra una RoI con la misma masa extraída de la proyección CC de la mama izquierda

Este artículo presenta un método que clasifica automáticamente las masas como benignas o malignas contenidas en mamografías multi-vista. En lugar de llevar a cabo una segmentación completa de la lesión, la descripción automática se basa en una caracterización multi-resolución y multi-escala de la región de interés (RoI) extraída de al menos dos proyecciones MLO y CC, así cada RoI es proyectada sobre dos espacios de transformación diferentes definidos, el primero por los momentos de Zernike y el segundo por la transformada Curvelet, respectivamente. Finalmente, esta información heterogénea es fusionada mediante una estrategia de aprendizaje de múltiples kernels (Multiple Kernel Learning) construida por un entrenamiento con máquinas de soporte vectorial (SVM). En trabajos anteriores se ha mostrado el potencial de las características 
de multi-resolución como descriptores de masas en una proyección mamográfica [33, 34, 35] y en este artículo se amplía estos trabajos generalizando la representación y aprendiendo de las características relevantes extraídas de cada proyección para finalmente correlacionar dicha información, logrando así construir un único descriptor extraído de las dos proyecciones.

Las principales contribuciones de este trabajo se resumen a continuación:

1. Una nueva caracterización de masas mamográficas tomadas de dos proyecciones diferentes y que fusiona dos bases complementarias de descomposición multi-resolución, el análisis piramidal de los momentos de Zernike, una base ortogonal compleja y la transformada Curvelet, una descomposición multi-escala adecuada para capturar mezclas complejas de líneas curvas.

2. Un método que integra varias proyecciones del mismo hallazgo mamográfico para clasificar su nivel de severidad (maligno/ benigno) acorde al protocolo clínico real radiológico.

El resto de este artículo está organizado de la siguiente manera: en la siguiente sección se resume una revisión de la literatura de trabajos previos desarrollados para clasificar masas según un análisis ipsilateral. En la sección 3, se describe el método propuesto. La sección 4 presenta los resultados experimentales, y la última sección está dedicada a discusiones y conclusiones.

\section{Materiales y métodos}

En la figura 3 se ilustra el esquema de trabajo del método propuesto. Inicialmente, el radiólogo experto selecciona, de forma manual, una masa, la misma que está contenida en las dos vistas mamográficas (MLO y CC) de la misma mama, como Regiones de Interés, entonces la estrategia comienza aplicando una etapa de pre-procesamiento para cada una de las Regiones de Interés seleccionadas, una vez que se han mejorado algunos detalles visuales, la información correspondiente a la forma y textura de la masa, es extraída usando los momentos de Zernike y la transformada Curvelet. Esta información es combinada usando una estrategia de aprendizaje por 
múltiples kernels (Multiple Kernels Learning) asignando pesos según la relevancia de las características visuales en tareas de clasificación, un procedimiento que se logra mediante un entrenamiento basado en máquinas de soporte vectorial (SVM). Una vez que las regiones son caracterizadas, estas son clasificadas como benigna o maligna.

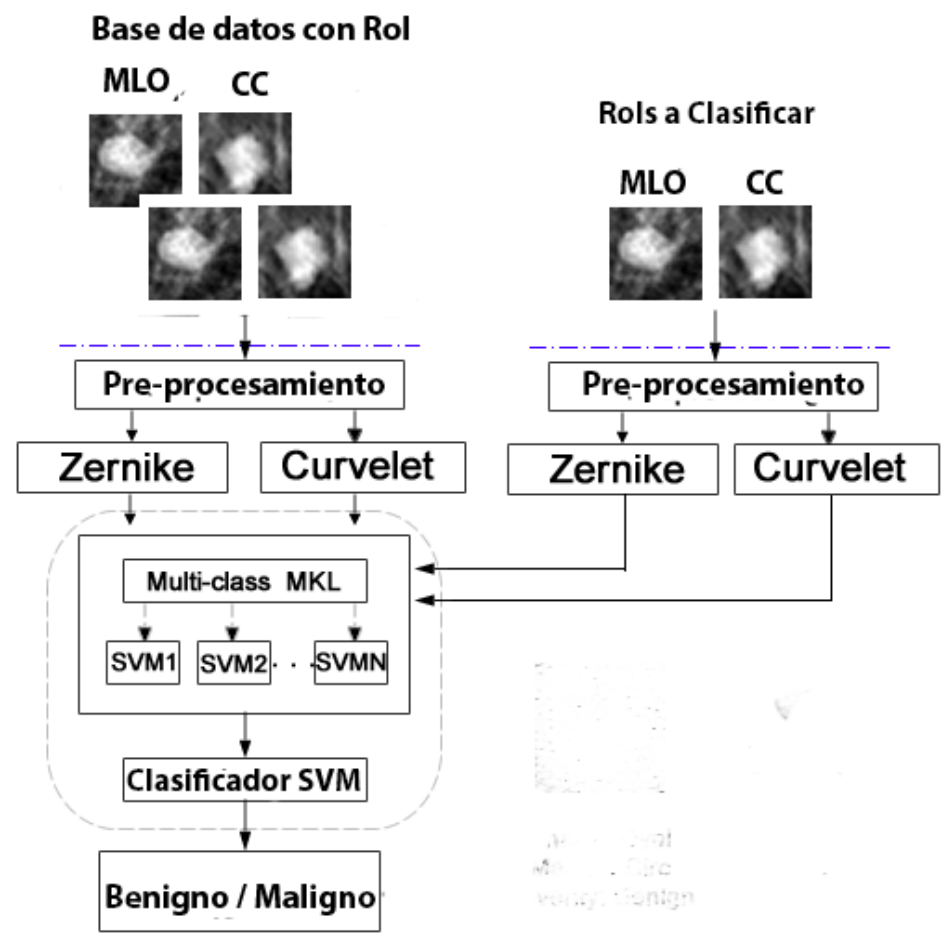

Figura 3. Esquema general de la estrategia propuesta

\section{A. Preprocesamiento de mamografías}

Inicialmente, las mamografías usadas en este trabajo fueron normalizadas mediante una estrategia de conversión de escala de grises hasta los 8-bits de profundidad [36]. Para este efecto, únicamente se seleccionaron mamografías que contenían algunas masas con un diámetro estimado hasta de $2 \mathrm{~cm}$, las mismas que corresponden a una región cuadrada (RoI) de $512 \times 512$ pixeles, las 
cuales son más probables a desarrollar cáncer en tapas tempranas y mucho más difíciles de diagnosticar [37]. Fundamentalmente, se ha demostrado que los radiólogos integran la información visual percibida a varias escalas y diferentes frecuencias [38]. En el caso particular de las masas, el análisis mamográfico integra información de los bordes y patrones espaciales locales en diferentes escalas y a diferentes orientaciones. Inspirado en estos estudios, en el presente trabajo se ha explorado dos representaciones complementarias que describen las lesiones mamográficas de manera global (descriptor de forma) y de manera local (descriptor de textura). Para el primer caso, se propone un análisis piramidal de los momentos de Zernike con el propósito de capturar información esencial correspondiente a la forma de la masa y una descomposición mediante Curvelets a diferentes escalas y orientaciones para capturar información de diferentes lineas curvas y patrones de textura, pero para cada descriptor fue necesario un pre-procesamiento diferente, los mismos que se describirán a continuación:.

\section{B. Descriptor de forma de masas: análisis por los momentos de Zernike}

La forma de las masas ha sido reportada entre los signos radiológicos más importantes [39, 6, 40] y los momentos de Zernike se han usado clásicamente como buenos descriptores para caracterizar formas [20, 22]. Los momentos de Zernike se derivan de un conjunto de polinomios ortogonales complejos, proyectados dentro del círculo unitario [41, 42], los mismos que son expresados en coordenadas polares y calculados desde un conjunto de polinomios de Zernike, tal como:

$$
V_{p, q}(r, \theta)=R_{p, q}(r) e^{j q \theta}, r \in[-1,1]
$$

donde, $p$ representa el orden del polinomio y $q$ sus repeticiones (frecuencias). Así, el orden $p$ es un interno no negativo y la repetición $q$ es un entero positivo o negativa, de tal manera que satisface la condición $p-|q|$ es un número par y $|q| \leq p$. Los polinomios radiales con valores reales están dados por: 


$$
R_{p, q}(r)=\sum_{k=q}^{p} B_{p|q| k} r^{k} e^{j q \theta}
$$

donde, $r=\sqrt{x^{2}+y^{2}}$ es la magnitud del vector $\theta=\tan ^{-1}\left(\frac{y}{x}\right)$ y su ángulo, y sus coeficientes $B_{p|q| k}$ están dados por:

$$
B_{p|q| k}=\frac{(-1)^{\frac{p-k}{2}}\left(\frac{p+k}{2}\right) !}{\left(\frac{p-k}{2}\right)\left(\frac{k+q}{2}\right) !\left(\frac{k-q}{2}\right) !}
$$

Estas funciones ortogonales, permiten descomponer una imagen $f(x, y)$ como una suma ponderada de los polinomios de Zernike, tal como: $\sum_{p=1}^{\infty} \sum_{q=-p}^{p} Z_{p, q} V_{p, q}$, donde $Z_{p, q}$ son los momentos de Zernike de orden p, con q repeticiones. Esta relación expresada en términos discretos, en donde una imagen digital de tamaño $N \times N$ es proyectada dentro del espacio de Zernike contenido por todas las funciones polinomios ortogonales, se presenta como:

$$
Z_{p q}=\frac{p+1}{\lambda} \sum_{x=0}^{N-1} \sum_{y=0}^{N-1} V_{p q}^{*}(x, y) f(x, y)
$$

Donde, $\lambda=\delta \mathrm{A} / \pi$ es una constante de normalización y $\delta \mathrm{A}$ es el área de la imagen cuando es proyectada sobre el circulo unitario de los polinomios de Zernike.

Como un primer paso, se aplicó el método propuesto en [35], para resaltar las características de forma de la masa, el cual consistió en cuantificar el rango dinámico del histograma de nivel de grises original en 12 bins dentro del intervalo de [0,255]. Para tal efecto, se asigna una RoI particular al círculo unitario haciendo coincidir el centroide de la RoI y el círculo unitario. De esta manera, se calcularon los momentos de Zernike de $45^{\text {th }}$ hasta el $50^{\text {th }}$ orden, siendo estos valores los suficientes para reconstruir la forma original con una relación-señal a ruido pico, PSNR (Peak Signal-to-Noise Ratio), 
mayor a 40:dB. Del mismo modo, este orden seleccionado evita cualquier inestabilidad numérica producida por la presencia de altas frecuencias de la imagen, tal como se ha demostrado en [35], siendo esta una limitación de los polinomios de Zernike producidos por la discretización de estas bases continuas. En este trabajo los errores numéricos y geométricos producidos por el uso de esta estrategia se corrigieron como se muestra en [42]. Finalmente, este principio de extracción de características se aplicó para las dos regiones desde las vistas MLO y CC, para finalmente construir un vector de características general mediante la concatenación del histograma normalizado de acuerdo a las frecuencias (repeticiones) para cada uno de los cinco órdenes (45 a 50) de los momentos de Zernike, obtenidos por cada RoI en las diferentes vistas.

Por otro lado, los efectos producidos por la invarianza a la traslación y escalado de la imagen se evitan mediante la aplicación de una técnica de normalización como se describe en [43], al centrar y escalar el marco de referencia $(x, y)$ a $\left(\frac{x}{a}+\bar{x}, \frac{y}{a}+\bar{y}\right)$, siendo $(\mathrm{x}, \overline{\mathrm{y}})$ el centroide $f(x, y)$ y $a=\sqrt{\beta / m_{00}}$ el factor de escala, con $\beta$ el número de pixeles en la masa obtenida al binarizar el RoI y $m_{00}$ el momento geométrico de orden cero.

Clásicamente, las representaciones de formas tomadas desde los primeros cinco órdenes de los polinomios de Zernike han sido usados como una aproximación a las aberraciones ópticas producidas por el ojo humano $[19,18]$, ignorando los coeficientes de orden alto ya que, dependiendo del tamaño de la imagen, un cálculo simple para los primeros 40 momentos pueden tomar una hora [42]. Sin embargo, con la estrategia propuesta en este trabajo, el costo computacional se reduce drásticamente en la fase de pre-procesamiento, los momentos hasta el orden cincuenta pueden calcularse en menos de 5s. Además, este subconjunto de momentos también se seleccionó para obtener una representación de las características globales de masas (ver figura 4). 


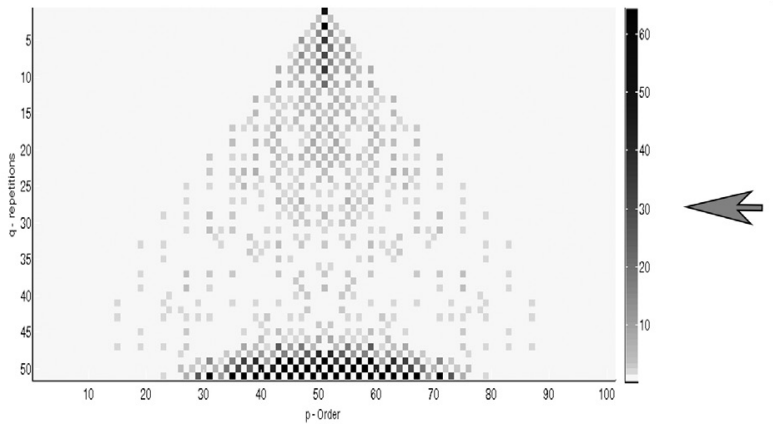

(a)

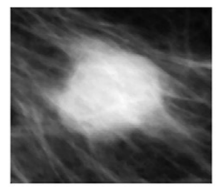

Original Rol

(b)

Figura 4. Descomposición piramidal mediante los momentos de Zernike (frecuencia vs. orden): a) Ilustra los coeficientes de Zernike hasta el orden 50 de la imagen original. b) Ilustra una RoI con una masa extraída de la proyección $\mathrm{CC}$

\section{Caracterización por la transformada Curvelet}

La transformada Curvelet realiza una descomposición multiescala [44] utilizando un conjunto de bases estadísticamente dependientes. En donde cada RoI de las proyecciones MLO y CC, son descompuestas para capturar su información de acuerdo a diferentes escalas, de igual manera, cada una de estas bases Curvelet captura su orientación asociada. Esta transformación, en particular, limita cada escala mediante la ley de escalamiento anisotrópico parabólico [44], asegurando que la forma de masa y los patrones de textura se conserven a diferentes escalas. Varias investigaciones han demostrado que Curvelet es un descriptor de textura adecuado en diferentes bases de datos públicas con imágenes de textura [45], mientras que otras han demostrado que Curvelets supera las transformadas como wavelets o Gabor en tareas de clasificación [32, 46]. En el dominio de la frecuencia, un Curvelet se construye como el producto de dos ventanas: una angular y las coronas radiales diádicas. La ventana angular representa el análisis direccional y la ventana diádica radial es un filtro pasa-banda con frecuencias de corte que siguen la ley de escalado anisotrópico parabólico widh $\approx$ length ${ }^{2}$ [44]. Debido a esta propiedad anisotrópica, los Curvelets se adaptan mucho mejor a 
las curvas a diferentes escalas que otras transformaciones y, por lo tanto, resultan muy resistentes al ruido en las imágenes. La figura 5 ilustra la descomposición mediante la transformada Curvelet de una RoI.

El pre-procesamiento para este caso particular de extracción de características, busca preservar los patrones principales de textura y definir un nivel comparable de intensidad, para ello, un filtro basado en la mediana fue aplicado, suavizando la RoI de cada proyección (MLO y CC), mientras que con un ajuste adaptativo del histograma [47] se lograron resaltar los patrones de textura principales y conserva los bordes importantes, como se ilustra en la figura 6 .

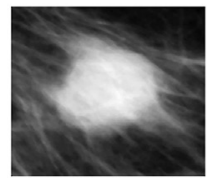

Original Rol

(b)

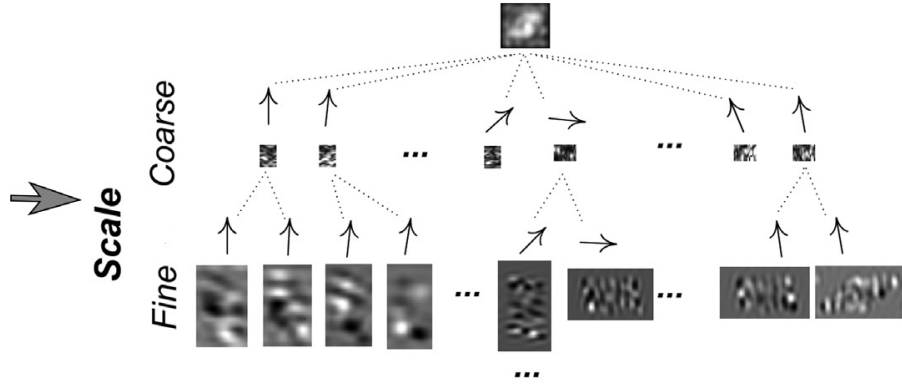

(c)

Figura 5. Descomposición multi-resolución mediante la transformada Curvelet de una RoI a diferentes escalas y orientaciones

Luego de que las dos regiones de interés RoI de las dos proyecciones fueron descompuestas en cuatro escalas con 32 orientaciones, para un total de 128 sub-bandas. El descriptor basado en curvelets se construyó concatenando versiones normalizadas de los coeficientes curvelets extraídos de las diferentes subbandas, es decir, 32 orientaciones en las cuatro escalas por la RoI de la proyección MLO y la región de la proyección CC. Para este caso particular y con el propósito de definir la similitud para este tipo de descripción, se ha implementado una medida de similitud basada en la Intersección de Histogramas. La transformada Curvelet fue implementada mediante 
la transformada rápida de Fourier desigualmente espaciada, con una ventana rectangular inclinada a lo largo de la dirección principal de cada curvelet, como es presentado en [44]

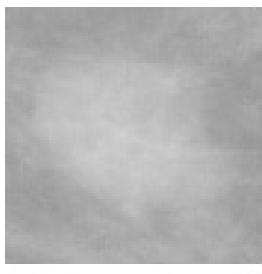

(a)

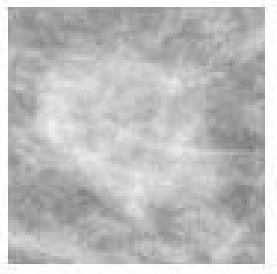

(b)

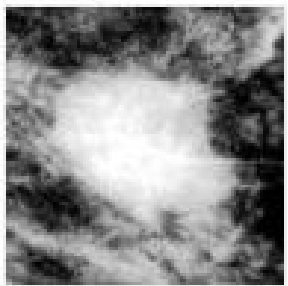

(c)

Figura 6. Etapa de procesamiento mediante un filtro de mediana y ajuste adaptativo del histograma. a) Ilustra una RoI original con una masa, b) Ilustra una RoI procesada con un filtro de mediana, c) Ilustra la RoI mejorada por ajuste adaptativo del histograma

\section{Fusión de descriptores multiresolución}

Una vez que se realizaron los análisis de Zernike y Curvelet para cada RoI (MLO y CC), se aplica una estrategia de fusión de información con el propósito de encontrar una forma óptima de combinar la información obtenida de estas dos fuentes de representación. Específicamente, la fusión propuesta intenta encontrar valores óptimos para los parámetros que maximizan la capacidad discriminativa para las clases maligna y benigna de una masa. Una estrategia común es ponderar los espacios de características (descriptores Zernike y Curvelets) y luego sumarlos para calcular una caracterización óptima, tal como [48]:

$$
\hat{S}(x)=\sum_{\phi} \omega_{\phi} S^{\phi}(x)
$$

El problema con esta estrategia es que la información importante de las características individuales puede perderse cuando se com- 
binan de manera lineal los espacios de características. Por lo tanto, la fusión propuesta en este trabajo usa una estrategia diferente que mantiene toda la información de los dos espacios de características y solo los fusiona cuando se necesita tomar una decisión. Específicamente, los espacios de Zernike y Curvelet se utilizan como entrada para una función discriminante, $D W(x)$, que indica a qué espacio pertenece un determinado valor de $\mathrm{x}$ para una clase en particular:

$$
D_{W}(x)=\left\langle W,\left(\Psi_{1}\left(S^{1}(x)\right), \ldots, \Psi_{\Phi}\left(S^{|\Phi|}(x)\right)\right)\right\rangle
$$

Donde, $\mathrm{W} \in \mathrm{R}^{|\Phi| \times \mathrm{M} \times \mathrm{N}}$ es el vector de parámetros que indica la importancia relativa del vector de características extraído de cada espacio $S^{\varphi}(x)$ y $\Psi_{1, \ldots, \phi}:\left[\mathrm{S}^{\phi}\right]^{\mathrm{M} \times \mathrm{N}} \rightarrow F$ es la función que mapea cada descriptor $S^{\varphi}(x)$ para un espacio de características $\mathrm{F}$. El valor de $D_{W}(x)$ es esperado a satisfacer $D(x) \geq 0$ sí $x$ corresponde a una específica clase y $D_{W}(x)<0$ sí $x$ no es parte de esa clase. Esta formulación tiene la ventaja de mejorar la flexibilidad permitiendo que el modelo tenga en cuenta interacciones no-lineales potencialmente complejas entre los valores de un espacio único original correspondiente a características en diferentes escalas.

Considerando que solo dos espacios de características diferentes deben ser óptimamente fusionados, dos funciones $\Psi$, específicamente la función $\Psi_{1}$ y $\Psi_{2}$ son encargadas de mapear los descriptores a un nuevo espacio de características, donde los patrones complejos no-lineales se vuelven lineales. Esta estrategia es bien conocida como kernel trick y es usada con métodos de kernels, donde el mapeo $\Psi_{1}$ y $\Psi_{2}$ es implícitamente inducida por una función kernel. Así, una función kernel $k: X \times X \rightarrow R$ asociado a un mapeo $\Psi_{1, \ldots, \phi}: \mathrm{X} \rightarrow \mathrm{F}$ tal que $\forall \mathrm{x}, \mathrm{y} \in \mathrm{X}, \mathrm{k}(\mathrm{x}, \mathrm{y}) \leq \Psi_{1}(\mathrm{x}), \Psi_{1}(\mathrm{y})>F$, i.e., $k$ calcula el producto punto en $F$. De esta manera, un Kernel puede ser visto como una función que mide la similitud entre dos objetos desde un espacio de características. En este trabajo, el espacio de características de entrada puede ser el espacio de las Curvelets y los momentos de Zernike que son fusionados, entonces la función kernel mide la similitud en esos espacios particulares.

Esto es formulado como problema de maximizar y minimizar el siguiente problema de discriminación: 


$$
\min _{W} C \sum_{i=1}^{N} \max \left(0,1-y_{i} D_{W}\left(x_{i}\right)\right)+\|W\|_{2}^{2}
$$

donde, $x_{i}$ representa una RoI de entrenamiento, $\mathrm{y}_{\mathrm{i}} \in\{-1,1\}$ representa la clase correspondiente, $N$ es el número de muestras usadas en el entrenamiento, y $C$ controla la regularización del modelo. El primer término de la función objetivo en la ecuación 7 es una función de pérdida que penaliza la clasificación incorrecta de las muestras de entrenamiento y el segundo término es el regularizador del parámetro $W$. La regularización de $W$ está asociada con la búsqueda de un máximo margen del clasificador de la misma manera como funciona para la clasificación mediante máquinas de soporte vectorial [49]. En este trabajo, el parámetro C fue de 1000 después de una búsqueda exhaustiva del valor óptimo bajo una estrategia de entrenamiento mediante una validación cruzada con 10 sub-grupos de datos, conocida como "10-fold cross validation".

Entonces, considerando las funciones $\Psi_{1}$ y $\Psi_{2}$, en este trabajo se usó la intersección de histogramas como una función kernel, la cual es definida como:

$$
\Psi_{1}=k_{h i}\left(s_{p}, s_{q}\right)=\sum_{i} \sum_{j} \min \left(s_{p}(i, j), s_{q}(i, j)\right)
$$

lo cual requiere que los momentos de Zermike sean normalizados entre valores de 0 a 1 . Así, el modelo propuesto fusiona los diferentes espacios combinando sus respectivos kernels, en lugar de directamente sumar los mismo espacios. El problema de optimización en la ecuación 7 es por lo tanto tratado como un problema de aprendizaje basado en múltiples kernels, conocido como Multiple Kernel Learning (MKL), con el cual se busca un buen hiper-plano discriminante en el espacio fusionado. Varias formulaciones basadas en MKL han sido desarrolladas últimamente, la estrategia usada en este trabajo se basa en la propuesta por [50], Aprendizaje Generalizado basado en Multiples Kernel (GMKL). 


\section{E. Clasificación de masas}

Esta etapa de clasificación consiste en establecer sí una nueva masa seleccionada por el radiólogo, en las dos vistas (MLO y CC), corresponde a una masa maligna o benigna de acuerdo a los criterios establecidos por el sistema BI-RADS. Una vez que las RoIs se asignan a los dos espacios diferentes como se describió hasta ahora, las diferentes representaciones alimentan un clasificador binario SVM (Support Vector Machine) que utilizan sus kernels pre-calculados basados en la intersección del histograma como medida de distancia. Este clasificador para discriminar entre las dos clases, la clase benigna y maligna, asume como valores positivos y negativos para cada clase respectivamente, lo cual es determinado por el signo de la siguiente función discriminante [51]:

$$
f_{i}=\frac{\mathbf{w}_{i} \cdot x+\mathbf{b}_{i}}{\left\|\mathbf{w}_{i}\right\|}, i=1,2, \ldots, n
$$

donde $w_{i}$ y $b_{i}$ son la normal y el peso hacia el hiper-plano relacionado a el $i^{\text {th }}$ clasificador. Por lo tanto, dada las dos RoIs $x$, el conjunto de clase $c_{i}=$ \{benign,malign $\}$. es asignado de acuerdo al sign: $\left(f_{i}(x)\right)$

\section{F. Medidas de Evaluación}

En tareas de clasificación, el clasificador debe asignar una_etiqueta (clase) de una colección de posibilidades finita y mutuamente exclusivas y, por lo tanto, cada instancia estará asociada a una sola clase. Esto generalmente se encuentra en la literatura como el método de relevancia binaria [51] por lo que se han utilizado diferentes medidas de desempeño. En este trabajo, el desempeño es evaluado en términos de la exactitud (accuracy), sensibilidad, especificidad y el área bajo la curva (AUC) de la curva RoC (Receiver Operating Characteristic) que es una representación gráfica de la sensibilidad frente a la especificidad para un sistema clasificador binario de acuerdo el umbral de discriminación. Estas medidas son definidas como: 


$$
\text { Accuracy }=\frac{T P+T N}{T P+T N+F P+F N}
$$

$$
\begin{gathered}
\text { Sensibilidad }=\mathrm{TPR}=\frac{T P}{T P+F P} \\
\text { Especificidad }=\frac{T N}{T N+F N}
\end{gathered}
$$

donde $T P, T N, F P$ y $F N$, denotan la cantidad de verdaderos positivos, falsos negativos, falsos positivos y falsos negativos, respectivamente, obtenidos de dicha tarea de clasificación. En consecuencia, se entrenó y evaluó el esquema de clasificación SVM, para el cual se determinaron los parámetros de clasificación durante la etapa de entrenamiento mediante una validación cruzada con 10 particiones de datos (10-fold cross validation).

\section{G. Descripción de las bases de datos}

La estrategia propuesta es evaluada utilizando diferentes niveles de calidad de imágenes mamográficas, una versión digitalizada de mamografías de placa (screen-film) y mamografías digitales de campo completo. Para esto, se usan dos bases de datos diferentes de dominio público, la Base de datos digital para la detección de mamografías $(D D S M)^{1}$ y la base de datos INBreast ${ }^{2}$, de las cuales fueron extraídos dos conjuntos de RoIs de las vistas MLO y CC, respectivamente. Una breve descripción para cada conjunto de datos se muestra debajo.

- Base de datos DDSM: la base de datos digitalizada para tamizaje de mamografía (DDSM) [52] es una base de datos con imágenes mamográficas ampliamente usada en esta área de investigación como punto de referencia de evaluación [53, 54, 55]. Esta base de datos de libre acceso está constituida por imágenes digitalizadas de películas mamográficas con su infor-

1 http://marathon.csee.usf.edu/Mammography/Database.html

2 http://medicalresearch.inescporto.pt/breastresearch/index.php/Get_INbreast_ Database 
mación técnica y diagnóstico clínico correspondiente. Toda la base de datos DDSM contiene un total de 2620 casos, cada uno incluye cuatro imágenes obtenidas de las vistas Cranio-Caudal (CC) y Mediolateral-Oblicua (MLO), así como una descripción específica de acuerdo al sistema BI-RADS, y que fueron anotadas por radiólogos expertos según BI-RADS cuarta edición. En esta investigación, se eligió un subconjunto de mamografías, las mimas que cumplieron los siguientes criterios de inclusión: 1) similar calidad de imagen, 2) número equilibrado del tipo de masas (benignas/malignas), 3) solo se evaluó una sola lesión, es decir, este estudio excluyó masas con calcificaciones, distorsión arquitectural o casos con la presencia de clips o marcas de metal en el área de masa. Por lo tanto, fueron seleccionadas un subconjunto de mamografías digitalizadas con un escáner láser Lumisys a $50 \mu \mathrm{m}$ o un escáner Howtek a una resolución de $43.5 \mu \mathrm{m}$ píxeles y con un rango dinámico de intensidades de $=4096$ niveles de tono de grises. Luego, un proceso de extracción de la RoI fue llevado a cabo. Para lo cual se consideró la información relacionada a la ubicación y tamaño de la masa de cada caso, permitiendo recortar manualmente subimágenes cuadradas considerando como centro las masas. Debido a que las anotaciones proporcionadas en la base de datos DDSM incluyen el límite de la segmentación de los hallazgos, en este trabajo el tamaño de las RoIs se definió como un cuadrado de nxn pixeles. Específicamente, las RoI se recortaron según el cuadro delimitador de las lesiones y se redimensionaron a nxn píxeles conservando la relación de aspecto cuando el ancho o la altura del cuadro delimitador son mayores que n; en otros casos, la lesión se centra sin escalar y se preservan los tejidos de fondo. En consecuencia, un conjunto de 980 regiones de interés fueron extraídas de esta base de datos DDSM incluyendo vistas CC y MLO, distribuidas como 490 regiones de interés con masa de la vista MLO y 490 regiones de interés de la vista CC de la misma mama.

- Base de datos INBreast: una evaluación adicional fue llevada a cabo usando los casos almacenados en la base de datos INBreast [56]. Esta base de datos contiene mamografías en 
formato DICOM y fueron adquiridas del Hospital de São João, Breast Centre, Porto, Portugal. Esta colección de casos incluye masas, calcificaciones, asimetrías bilaterales y distorsión arquitectural. En este trabajo, se seleccionaron un total de 108 cases (216 imágenes) que constituyen esta base de datos, de los cuales 108 casos son documentados ipsilateralmente (4 imágenes por caso) y 25 son de pacientes con mastectomía (2 imágenes en estos casos). En consecuencia, un conjunto de 108 masas fueron extraídas por cada vista MLO y CC. La localización y el tamaño de las masas, asociadas a cada caso, fueron tomados de los contornos dibujados por los expertos y proporcionados como información en formato XML.

\section{Resultados}

El método propuesto fue evaluado dos veces. Primero, para la tarea de clasificar RoIs de entrada tomadas de la base de datos DDSM y determinar sí corresponde a la clase benigna o maligna. En segundo lugar, la evaluación fue llevada a cabo con las RoIs tomadas de la base de datos INBreast para la misma tarea de clasificación. En ambos casos, se evaluó el desempeño del método de fusión de información de las dos proyecciones (MLO y CC) respecto a la clasificación obtenida por cada vista. El proceso de evaluación experimental fue implementado en MATLAB R17, ejecutado en una PC Linux con 2 Intel Quad Core i7 a 3.07 GHz y 24 GB de RAM. Todos los experimentos fueron implementados y basados en el código fuente de Generalized Multiple Kernel Learning (GMKL), así como, la implementación de la estrategia Multiple Kernel Learning [50], mientras que el clasificador SVM fue implementado usando la librería de código abierto LIBSVM [57], con matrices de distancia como kernels pre-calculados.

\section{A. Base de Datos DDSM}

La primera evaluación del método consistió en comparar el poder discriminante del esquema de fusión de información obtenida de las dos proyecciones MLO y CC frente a la caracterización de la misma masa únicamente vista en cada proyección por separado, para tal efecto, una misma masa fue caracterizada mediante los 
espacios de Zernike y Curvelets en la proyección MLO y CC. Esta evaluación permitió determinar el rendimiento del esquema de fusión propuesto frente a imágenes con calidad variable, como es el caso de las imágenes de la base de datos DDSM. En esta evaluación se usaron 980 RoIs extraídas de la base de datos DDSM (490 MLO y 490 CC). Por lo tanto, en esta evaluación se comparó el desempeño obtenido con la proyección medio lateral oblicua (MLO), proyección cráneo caudal (CC) y la estrategia de fusión de las dos proyecciones (MLO-CC). Las dos espacios de características se usaron como entradas para un clasificador SVM binario clásico con matrices de kernel pre-calculadas. Para el proceso de entrenamiento se llevó a cabo una partición de 70-30 muestras, para entrenamiento se seleccionó el $70 \%$, mientras que el 30\% restante se usó para la validación, un proceso que fue repetido 10 veces. Los resultados obtenidos durante esta evaluación se presentan en la Tabla 1.

Tabla 1. Rendimiento promedio según un esquema de validación de 70-30\% para la tarea de clasificar los RoI como benignas y malignas para las base de datos DDSM

\begin{tabular}{|c|c|c|c|}
\cline { 2 - 4 } \multicolumn{1}{c|}{} & \multicolumn{3}{c|}{ DDSM } \\
\cline { 2 - 4 } \multicolumn{1}{c|}{} & MLO-CC & MLO & CC \\
\hline Sensibilidad & 96.3 & 85.3 & 82.2 \\
\hline Especificidad & 93.1 & 83.2 & 80.4 \\
\hline AUC & 0.95 & 0.83 & 0.79 \\
\hline Accuracy & 97.8 & 86.1 & 80.3 \\
\hline
\end{tabular}

En general, la fusión de información correspondiente a las dos proyecciones (MLO-CC) supera al desempeño logrado por cada una de las proyecciones por separado MLO y CC, respectivamente, alcanzando una sensibilidad del $96.3 \%$ frente $85.3 \%$ y $82.2 \%$ correspondiente a la proyección MLO y CC, mientras las especificidades alcanzada de 93.1 fue superior a $83.2 \%$ y $80.4 \%$ alcanzados por cada proyección por separado. Además, las métricas Accuracy y AUC muestran resultados notables, con 97.8 y 0.95 para esa base de datos. Finalmente, estos resultados pueden ser observados mediante las curvas ROC, los cuales se ilustran en la figura 7 


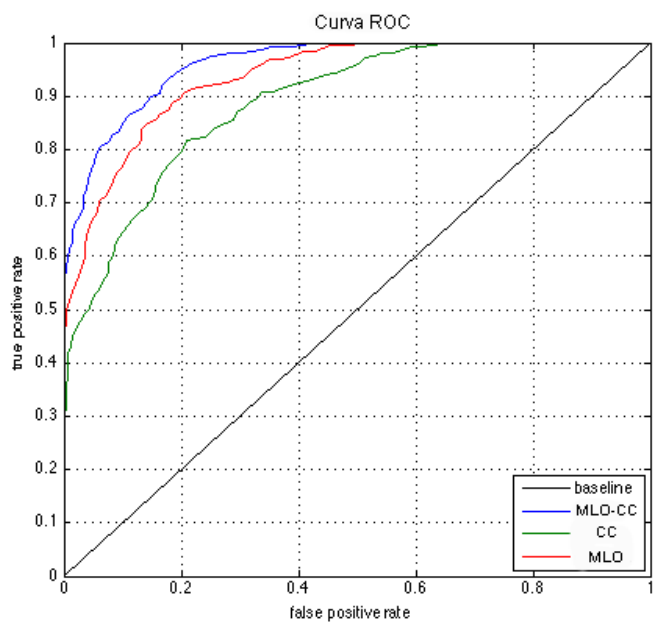

Figura 7. Curva ROC correspondiente al desempeño de la estrategia de fusión propuesta (MLO-CC) respecto a la clasificación usando información de una única vista MLO y CC, respectivamente

\section{B. Base de Datos INBreast}

En esta evaluación, se midió el desempeño de la estrategia propuesta en imágenes digitales DICOM con la misma calidad. De igual manera, esta evaluación consistió en comparar el poder discriminante del esquema de fusión de información obtenida de las dos proyecciones MLO y CC frente a la caracterización de la misma masa únicamente vista en cada proyección por separado. En esta evaluación se usaron 216 RoIs extraídas de la base de datos INBreast (108 MLO y 108 CC). De igual manera, se comparó el desempeño obtenido con la proyección medio lateral oblicua (MLO), proyección cráneo caudal (CC) y la estrategia de fusión de las dos proyecciones (MLO-CC). Las dos espacios de características se usaron como entradas para un clasificador SVM binario clásico con matrices de kernel pre-calculadas. Para el proceso de entrenamiento se llevó a cabo una partición de 70-30 muestras, para entrenamiento se seleccionó el 70\%, mientras que el 30\% restante se usó para la validación, un proceso que fue repetido 10 veces. Los resultados obtenidos durante esta evaluación se presentan en la Tabla 2. 
Tabla 2. Rendimiento promedio según un esquema de validación de 70-30\% para la tarea de clasificación de RoIs con masas benignas y malignas de la misma mama (base de datos INBreast)

\begin{tabular}{|c|c|c|c|}
\cline { 2 - 4 } \multicolumn{1}{c|}{} & \multicolumn{3}{c|}{ INBreast } \\
\cline { 2 - 4 } \multicolumn{1}{c|}{} & MLO-CC & MLO & CC \\
\hline Sensibilidad & 98.1 & 87.2 & 85.3 \\
\hline Especificidad & 95.6 & 83.4 & 81.2 \\
\hline AUC & 0.98 & 0.80 & 0.79 \\
\hline Accuracy & 97.2 & 85.3 & 82.7 \\
\hline
\end{tabular}

En este caso particular con la base de datos INBreast, los resultados reportados demuestran que la fusión de información de las dos proyecciones (MLO-CC) supera al desempeño logrado por cada una de las proyecciones por separado MLO y CC, respectivamente, alcanzando una sensibilidad del $98.1 \%$ frente $87.2 \%$ y $85.3 \%$ correspondiente a la proyección MLO y CC, mientras las especificidades alcanzada de 95.6 fue superior a $83.3 \%$ y $81.2 \%$ alcanzados por cada proyección por separado. Además, las métricas Accuracy y AUC muestran resultados notables, con 97.2 y 0.98 para esta base de datos. De igual manera que en el experimento anterior, los resultados de esta evaluación pueden ser visualizados mediante las curvas ROC, los cuales se ilustran en la figura 8.

\section{Discusión}

En este trabajo se ha propuesto un método novedoso que fusiona información visual completamente obtenida desde dos vistas diferentes de la misma masa para su clasificación entre benigna o maligna de acuerdo al criterio establecido en el sistema BI-RADS, comportándose como un modelo que se acerca al flujo de trabajo real de los radiólogos en escenarios clínicos reales, a la vez que cumple el requisito importante para ser implementado en cualquier sistema de soporte CAD. La importancia de esta estrategia se fundamenta en un par de descripciones complementarios multi-resolución, los mismos que se fusionan de manera óptima en un marco adaptable de aprendizaje basado en múltiples kernels. 


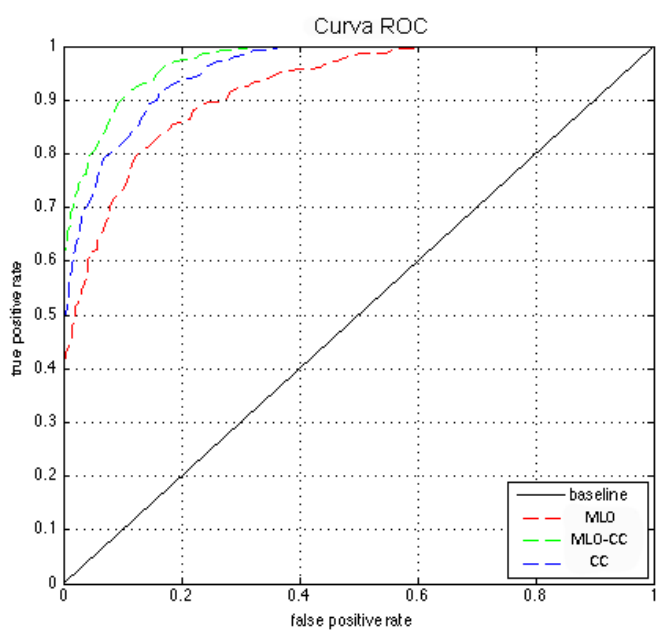

Figura 8. Curva ROC correspondiente al desempeño de la estrategia de fusión propuesta (MLO-CC) respecto a la clasificación usando información de una única vista MLO y CC, respectivamente

En este trabajo se aprovecharon las características visuales variables que pueden presentar entre una y otra proyección para refinar su clasificación, además de aprender del conocimiento implícito del radiólogo experto proporcionado sobre la anotación de la imágenes almacenadas en las bases de datos. La evaluación exhaustiva ha demostrado que el enfoque actual es altamente confiable para ser utilizado en escenarios clínicos reales y con cualquier estrategia de $\mathrm{CAD}$ para tareas de clasificación.

Frecuentemente, diferentes métodos para clasificar masas como benignas y malignas $[15,16,14,17]$, segmentan la lesión y extraen diferentes características cuyo rendimiento para esta tarea de clasificación se ha evaluado mediante las curvas ROC o FROC. Olivier et. Alabama. [17] proporcionó una comparación cuantitativa de siete métodos que comparten algunas estrategias básicas, tales como, crecimiento de regiones para localizar la presencia de estructuras específicas, cluster o agrupamientos de píxeles y modelos de detección de contornos específicos que caracterizan lesiones potenciales. Este conjunto de métodos se evaluó con una parte de la base 
de datos MIAS y una recopilación de datos privados, solo 261 casos que representan un conjunto de datos muy reducido, lo que reportaron áreas bajo la curva $\mathrm{ROC}$ de $\mathrm{AUC}=0.8$, sin embargo, el número de falsos positivos por imagen alcanzó más de cinco por cada casos. Por el contrario, el esquema propuesto basado en fusión de vistas MLO-CC fue evaluado con una base de datos compuesta por RoIs, extraídos de la base de datos DDSM y INBreast obteniéndose valores del AUC de 0.98 y 0.95 , respectivamente, en la tarea de clasificación como benigna y maligna. Así mismo por ejemplo, otros trabajos han utilizado diferentes análisis multi-resolución para construir un descriptor de bajo nivel. Tahmasbi et. al [18] han demostrado que una representación de forma útil, requiere una serie de momentos de Zernike [18, 19]. Así, evaluaron los momentos de Zernike calculados en un orden máximo (orden 17) y los dividieron en dos grupos, 32 de orden inferior y 32 de orden superior. La combinación de estos dos grupos resultó como el mejor descriptor de forma de las masas, reportando un AUC $=0.97$ en la tarea de clasificación como benignas o malignas, para lo cual, usaron una red neuronal como clasificador mediante un algoritmo Perceptron de múltiples capas. Este método fue evaluado con la base de datos MIAS, específicamente usaron, 209 ROI normales, 67 ROI benignos y 54 ROI malignos y la conclusión fue que los momentos de Zernike de bajo orden mostraron poca capacidad de discriminación, reportando un AUC $=0.59$, pero la combinación con órdenes superiores mejoró la resultados del método. En contraste, los resultados obtenidos en este trabajo superaron este método y fusionaron de manera óptima las representaciones de Zernike-Curvelet, con momentos de Zernike de orden de $45^{\text {th }}$ a $50^{\text {th }}$. Este conjunto de momentos fue seleccionado debido a que observamos que la mayor parte de la energía de los coeficientes se concentraba en este rango y, además, el PSNR durante la reconstrucción de la imagen original era mayor de 40: $d B$. De igual manera, Eltoukhy et. al [32] comparó las diferentes transformaciones wavelet y Curvelet para diferenciar cualquier tipo de lesión mamaria, específicamente, masas, calcificaciones, distorsión arquitectónica y asimetría bilateral. Para este propósito seleccionó un conjunto de 100 coeficientes de cada transformación y fueron usados con una estrategia de clasificación mediante los vecinos 
cercanos K-NN implementado con una métrica Euclideana. Para la validación se usaron 322 mamogramas, 51 malignos, 64 benignos y 207 normales. Las imágenes fueron descompuestas en cuatro niveles diferentes con tres funciones de wavelets diferentes, entre estas: Daubechies-8, symlet y bi-ortogonal, mientras que se usaron cuatro niveles de descomposición para las curvelets. Los resultados demostraron una tasa de clasificación promedio para las 3 clases (benigna, maligna y normal) de $94.07 \%$ para las Curvelet y $90.05 \%$ para las wavelets. Posteriormente, estos autores mejoraron sus resultados [32], utilizando un esquema SVM y logrando un $96.56 \%$ con la misma base de datos. Sin embargo, el análisis fue realizado en una única proyección de mamografías y su base de datos es mucho más pequeña que la utilizada en esta investigación, lo que se puede observar que el rendimiento es similar. Asimismo, se han reportado diferentes técnicas estadísticas, estructurales y espectrales para analizar las mamografías [24], incluidas las matrices de coocurrencia [25]. Ramos et.al [25] presentaron una evaluación de los métodos basados en Ridgelet, Wavelet, Co-ocurrence y concluyeron que la wavelet superó a las otras representaciones, con un AUC de 0.9 cuando clasificaba las masas como benignas o malignas, resultados que han sido mejorados con el método propuesto en este trabajo.

Una gran ventaja del presente método es que, es completamente automático, las evaluaciones mostraron un rendimiento notable en diferentes bases de datos, clasificando masas en regiones bien definidas y algunas con una pobre calidad de la imagen. La estrategia se basa en la fusión óptima de la información visual de dos vistas de la misma lesión y proveída por dos descripciones complementarias cuyos pesos se pueden aprender de la base de datos. El tamaño RoI se puede considerar un parámetro importante en los resultados y su influencia no se evaluó en este trabajo, sin embargo, el hecho de que los descriptores, Zernike y Curvelet, sean por naturaleza una descomposición multi-resolución y multi-escala podrían mitigar los efectos causados por la selección de este parámetro. Otro limitación del método se debe a la falta de detección automática de la masa (RoIs) en las dos proyecciones, en este caso las regiones se proporcionan como una entrada. Sin embargo, esto podría considerarse un inconveniente, pero el objetivo de esta investigación fue 
diseñar una estrategia integrable con un flujo de trabajo de radiólogo real y proporcionar alguna ayuda crucial al realizar tareas de diagnóstico. Se podría construir un método totalmente automático o de detección a partir de la presente estrategia mediante una simple búsqueda exhaustiva de toda la mamografía, pero esto estaba fuera del alcance de la presente investigación. Finalmente, el método muestra tiempos de ejecución asequibles del orden de 3-5 segundos.

\section{Agradecimientos}

Este trabajo fue parcialmente financiado por el Gobierno ecuatoriano a través de La Secretaría Nacional de Ciencia, Tecnología y Educación Superior (SENESCYT), Beca: 20110958 "convocatoria abierta 2011".

\section{Referencias}

[1] B. W. Stewart and C. P. Wild, "World cancer report 2014," 2014.

[2] M. Schneider, "Better detection: improving our chances, in: M.j. yaffe (ed.), digital mammography," in Digital Mammography, M. Yaffe, Ed., Fifth International Workshop on Digital Mammography. Toronto, Canada: Medical Physics Publishing, 2000, pp. 3-6.

[3] K. Kerlikowske, P. Carney, B. Geller, M. Mandelson, S. Taplin, K. Malvin,V. Ernster, N. Urban, G. Cutter, R. Rosenberg, and R. Ballard-Barbash, "Performance of screening mammography among women with and without a first-degree relative with breast cancer," Ann Intern Med.,vol. 133, no. 11, pp. 855-863, Dic 52000.

[4] M. Homer, Mammographic Interpretation: A Practical Approach, M.-H. S. Ed., Ed. New York, 1997.

[5] E. A. Sickles, C. J. DO' rsi, L. W. Bassett, and E. al., ACR BI-RADS Mammography. In: ACR BI-RADS Atlas, Breast Imaging Reporting and Data System., fifth edit ed. American College of Radiology, Reston, VA, 2013.

[6] L. J. Grimm, S. V. Ghate, S. C. Yoon, C. M. Kuzmiak, C. Kim, and M. A. Mazurows$\mathrm{ki}$, "Predicting error in detecting mammographic masses among radiology trainees using statistical models based on BI-RADS features." Medical physics, vol. 41, no. 3, p. 31909, mar 2014. [Online]. Available: http://scitation.aip.org/content/aapm/journal/ medphys/41/3/10.1118/1.4866379

[7] R. M. Nishikawa, "Current status and future directions of computeraided diagnosis in mammography," Computerized Medical Imaging and Graphics, vol. 31, pp. 224-235, 2007.

[8] B. Zheng, C. Mello-Thoms, X. Wang, G. Abrams, J. Sumkin, D. Chough, M. Ganott, A. Lu, and D. Gur, "Interactive computer aided diagnosis of breast 
masses: computerized selection of visually similar image sets from a reference library," Acad Radiol, vol. 14, pp. 917-927, 2007.

[9] D. Gur, J. Stalder, L. Hardesty, B. Zheng, J. Sumkin, D. Chough, B. Shindel, and H. Rockette, "Computer-aided detection performance in mammographic examination of masses: assessment." Radiology, vol. 233, pp. 418-423, 2004.

[10] L. A. L. Khoo, P. Taylor, and R. M. Given-Wilson, "Computer-aided detection in the united kingdom national breast screening programme: prospective study," Radiology., vol. 237(2), pp. 444-449, 2005.

[11] T. Freer and M. Ulissey, "Screening mammography with computer-aided detection: prospective study of 12,860 patients in a community breast center." Radiology, vol. 220, pp. 781-786, 2001.

[12] M. R. Rangayyana, F. J. Ayres, and J. L. Desautelsa, "A review of computer-aided diagnosis of breast cancer: Toward the detection of subtle signs," Journal of the Franklin Institute, vol. 344, pp. 312-348, 2007.

[13] N. Petrick, H. Chan, B. Sahiner, and D. Wei, "An adaptive density weighted contrast enhancement filter for mammographic breast mass detection," IEEE Trans. Med. Imaging, vol. 15, no. 1, pp. 59-67, 1996.

[14] H. Cheng, X. Shi, R. Min, L. Hu, X. Cai, and H. Du, "Approaches for automated detection and classification of masses in mammograms," Pattern Recognition, vol. 39, pp. 646-668, 2006.

[15] A. Oliver, J. Freixenet, J. Mart'1, E. P'erez, J. Pont, E. R. Denton, and R. Zwiggelaar, "A review of automatic mass detection and segmentation in mammographic images," Medical Image Analysis, vol. 14, pp. 87- 110, 2010.

[16] A. Tahmasbi, F. Saki, and S. B. Shokouhi, "Classification of benign and malignant masses based on zernike moments," Computers in Biology and Medicine, vol. 41, pp. 726-735, 2011.

[17] C.-H. Wei, S. Y. Chen, and X. Liu, "Mammogram retrieval on similar mass lesions," Computer Methods and programs in biomedicine, vol. 106, pp. 234-248, 2012.

[18] G. Papakostas, Y. Boutalis, D. Karras, and B. Mertzios, "A new class of zernike moments for computer vision applications," Information Sciences, vol. 177, no. 13, pp. 2802-2819, 2007.

[19] C. Wee and R. Paramesran, "On the computational aspects of Zernike moments," Image and Vision Computing, vol. 25, no. 6, pp. 967-980 , 2007.

[20] S. Li, M. C. Lee, and C. M. Pun, "Complex zernike moments features for shape-based image retrieval," IEEE Transactions on Systems, Man and Cybernetics, vol. 1, no. 39, pp. 227-237, 2009.

[21] C.-H. Wei, Y. Li, W. Chau, and C.-T. Li, "Trademark image retrieval: using synthetic features for describing global shape and interior structure of trademark," Pattern Recognition, vol. 42, no. 3, pp. 386-394, 2009.

[22] A. R. Dominguez and A. K. Nandi, "Detection of masses in mammograms via statistically based enhancement, multilevel-thresholding segmentation, and region selection," Computerized Medical Imaging and Graphics, vol. 32, pp. 304-315, 2008. 
[23] R. P. Ramos, M. Z. do Nascimento, and D. C. Pereira, "Texture extraction: An evaluation of ridgelet, wavelet and co-occurrence based methods applied to mammograms," Expert Systems with Applications, vol. 39, pp. 11 036-11 047, 2012.

[24] S. Nam and J. Choi, "A method of image enhancement and fractal dimension for detection of microcalcifications in mammogram," IEEE Engineering in Medicine and Biology Society, vol. 2, pp. 1009-1012, 1998.

[25] L. Bocchi, G. Coppini, J. Nori, and G. Valli, "Detection of single and clustered microcalcifications in mammograms using fractals models and neural networks," Medical Engineering and Physics, vol. 26, pp. 303-312, 2004.

[26] S. Liu, C. Babbs, and E. Delp, "Multiresolution detection of speculated lesions in digital mammograms," IEEE Transactions on Image Processing, vol. 10, no. 6, pp. 874-884, 2001.

[27] M. Z. do Nascimento, A. S. Martins, L. A. Neves, R. P. Ramos, E. L. Flores, and G. A. Carrijo, "Classification of masses in mammographic image using wavelet domain features and polynomial classifier," Expert Systems with Applications, vol. 40, no. 15, pp. 6213-6221, 2013.

[28] M. M. Eltoukhy, I. Faye, and B. B. Samir, "Breast cancer diagnosis in digital mammogram using multiscale curvelet transform," Computerized Medical Imaging and Graphics, vol. 34, pp. 269-276, 2010.

[29] F. Moayedi, Z. Azimifar, R. Boostani, and S. Katebi, "Contourlet-based mammography mass classification using the svm family," Comput. Biol. Med., vol. 40, no. 4, pp. 373-383, 2010.

[30] M. M. Eltoukhy, I. Faye, and B. B. Samir, "A comparison of wavelet and curvelet for breast cancer diagnosis in digital mammogram," Computers in Biology and Medicine, vol. 40, pp. 384-391, 2010.

[31] F. Narvaez, G. Diaz, and E. Romero, "Multi-view information fusion for automatic bi-rads description of mammographic masses," in Medical Imaging 2011: Computer-Aided Diagnosis, vol. 7963. International Society for Optics and Photonics, 2011, p. 79630A.

[32] — "Automatic BI-RADS Description of Mammographic Masses," in Digital Mammography, vol. 6136, 2010, pp. 673-681.

[33] F. Narv'aez, G. D'1az, F. G'omez, and E. Romero, "A content-based retrieval of mammographic masses using the curvelet descriptor," in Proc. SPIE, vol. 8315, 2012, pp. 83 150A-83 150A-7.

[34] F. Narv'aez, G. D'1az, C. Poveda, and E. Romero, "An automatic BIRADS description of mammographic masses by fusing multiresolution features," Expert Systems with Applications, vol. 74, pp. 82-95, 2017.

[35] A. A. AbuBaker, R. S.Qahwaji, M. J. Aqel, and M. H. Saleh, "Mammogram image size reduction using 16-8 bit conversion technique," International Journal of Medical, Pharmaceutical Science and Engineering, vol. 1, no. 3, pp. 83-90, 2007.

[36] D. Kopans, Brast Imaging, Philadelphia, Ed. Lippicott-Raven, 1998. 
[37] J. Beutel, H. Kundel, and R. V. Metter, Handbook of Medical Imaging, ser. Phys. Psychophys, S. Press, Ed. Bellingham, WA, 2000, vol. 1.

[38] A. C. of Radiology (ACR), "Illustrated breast imaging reporting and data system (bi-rads)," American College of Radiology, Reston, VA,, vol. third ed, p. 1998.

[39] C. Maggio, "State of the art of current modalities for the diagnosis of breast lesions," Eur. J Nucl Med Mol Imaging, vol. 31, no. 1, pp. S56-S69, 2004.

[40] S. X. Liao, "Image analysis with zernike moment descriptors," IEEE CCECE, pp. 700-703, 1997.

[41] K. M. Hosny, "Fast computation of accurate zernike moments," J Real-Time Image Proc, vol. 3, pp. 97-107, 2008.

[42] P. Dong and N. P. Galatsanos, "Affine transformation resistant watermarking based on image normalization," in Image Processing. 2002. Proceedings. 2002 International Conference on, vol. 3. IEEE, 2002, pp. 489-492.

[43] E. Candes, L. Demanet, D. Donoho, and L. Ying, "Fast discrete curvelet transforms," Multiscale Modeling and Simulation, vol. 5, no. 3, pp. 861-899, 2006.

[44] F. G'omez and E. Romero, "Texture characterization using a curvelet based descriptor," in Proceedings of the 14th Iberoamerican Conference on Pattern Recognition: Progress in Pattern Recognition, Image Analysis. Computer Vision, and Applications, 2009.

[45] M. Meselhy Eltoukhy, I. Faye, and B. Belhaouari Samir, "A statistical based feature extraction method for breast cancer diagnosis in digital mammogram using multiresolution representation." Computers in biology and medicine, vol. 42, no. 1, pp. 123-128, jan 2012.

[46] G. Kom, A. Tiedeu, and M. Kom, "Automated detection of masses in mammograms by local adaptive thresholding," Computers in Biology and Medicine, vol. 37, pp. 37-48, 2007.

[47] S. Frintrop, G. Backer, and E. Rome, "Goal-directed search with a topdown modulated computational attention system," Pattern Recognit, pp.117124, 2005.

[48] C. Cortes and V. Vapnik, "Support-vector networks," Machine Learning, vol. 20, no. 3, pp. 273-297, 2005.

[49] M. Varma and B. Babu, "More generality in efficient multiple kernel learning," in ACM 26th Annu. Int. Conf. Mach. Learn, 2009, pp. 1065- 1072.

[50] C.-W. Hsu and C.-J. Lin, "A comparison of methods for multiclass support vector machines," IEEE Transactions on Neural Networks, vol. 13, no. 2, pp. 415-425, 2002.

[51] M. Heath, K. Bowyer, D. Kopans, R. Moore, and W. P. Kegelmeyer, "The digital database for screening mammography," in Proceedings of the Fifth International Workshop on Digital Mammography, Medical Physics Publishing, vol. M.J. Yaffe, ed, pp. 212-218, 2001.

[52] D. C. Moura, M. A. Guevara L'opez, and M. A. G. L'opez, "An evaluation of image descriptors combined with clinical data for breast cancer diagno- 
sis." International journal of computer assisted radiology and surgery, vol. 8, no. 4, pp. 561-574, jul 2013

[53] A. Horsch, A. Hapfelmeier, and M. Elter, "Needs assessment for next generation computer-aided mammography reference image databases and evaluation studies," International Journal of Computer Assisted Radiology and Surgery, vol. 6, no. 6, pp. 749-767, 2011.

[54] X. Zhang, "A New Ensemble Learning Approach for Microcalcification Clusters Detection," Journal of Software, vol. 4, no. 9, pp. 1014-2021, 2009.

[55] I. C. Moreira, I. Amaral, I. Domingues, A. Cardoso, M. J. Cardoso, and J. S. Cardoso, "INbreast: toward a full-field digital mammographic database." Academic Radiology, vol. 19, no. 2, pp. 236-48, feb 2012.

[56] C.-C. Chang and C.-J. Lin., "LIBSVM : a library for support vector machines," ACM Transactions on Intelligent Systems and Technology, vol. 2, no. 27, pp. 1-27, 2011.

\section{Sobre el autor}

Fabián Rodrigo Narváez Espinoza. (Y'77-M’05). Director de la Carrera de Biomedicina. Se formó como Ingeniero Electrónico en la Universidad Politécnica Salesiana (2006). Obtuvo la maestría en Ingeniería Biomédica en la Universidad Nacional de Colombia (2010), con distinción Meritoria, y posteriormente el Doctorado en Ingeniería Mecánica y Mecatrónica, en la misma universidad (2016), con distinción Meritoria. Se desempeñó durante 8 años como Ingeniero de desarrollo en el Centro de Telemedicina y el Departamento de Imágenes Diagnósticas de la Universidad Nacional de Colombia-Sede Bogotá, con la inclusión de tecnologías emergentes para la prestación de servicios de medicina a distancia bajo la modalidad de Telemedicina. Durante el 2011 al 2015 fue docente-investigador en

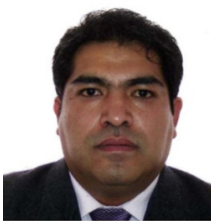
la Universidad Central (Bogotá). Actualmente, se encuentra vinculado como docente - investigador en la Universidad Politécnica Salesiana, Sede-Quito, en la carrera de Ing. Mecatrónica. Sus áreas de interés se enfocan en el procesamiento de señales biomédicas, reconocimiento de patrones, aprendizaje de máquina, visión por computador, procesamiento de imágenes diagnósticas y sistemas de diagnóstico asistido por computador. 